University of Wollongong

Research Online

Faculty of Engineering - Papers (Archive)

Faculty of Engineering and Information

Sciences

$1-1-2008$

\title{
Robust adaptive control of conjugated polymer actuators
}

Yang Fang

Michigan State University

Xiaobo Tan

Michigan State University

Gursel Alici

University of Wollongong, gursel@uow.edu.au

Follow this and additional works at: https://ro.uow.edu.au/engpapers

Part of the Engineering Commons

https://ro.uow.edu.au/engpapers/2870

\section{Recommended Citation}

Fang, Yang; Tan, Xiaobo; and Alici, Gursel: Robust adaptive control of conjugated polymer actuators 2008, 600-612.

https://ro.uow.edu.au/engpapers/2870

Research Online is the open access institutional repository for the University of Wollongong. For further information contact the UOW Library: research-pubs@uow.edu.au 


\title{
Robust Adaptive Control of Conjugated Polymer Actuators
}

\author{
Yang Fang, Student Member, IEEE, Xiaobo Tan, Member, IEEE, and Gürsel Alici
}

\begin{abstract}
Conjugated polymers are promising actuation materials for bio- and micromanipulation systems, biomimetic robots, and biomedical devices. Sophisticated electrochemomechanical dynamics in these materials, however, poses significant challenges in ensuring their consistent, robust performance in applications. In this paper, an effective adaptive control strategy is proposed for conjugated polymer actuators. A self-tuning regulator is designed based on a simple actuator model, which is obtained through reduction of an infinite-dimensional physical model and captures the essential actuation dynamics. The control scheme is made robust against unmodeled dynamics and measurement noises with parameter projection, which forces the parameter estimates to stay within physically meaningful regions. The robust adaptive control method is applied to a trilayer polypyrrole (PPy) actuator that demonstrates significant time-varying actuation behavior in air due to the solvent evaporation. Experimental results show that, during 4-h continuous operation, the proposed scheme delivers consistent tracking performance with the normalized tracking error decreasing from $11 \%$ to $7 \%$, while the error increases from $\mathbf{7 \%}$ to $28 \%$ and to $50 \%$ under a proportional-integral-derivative (PID) controller and a fixed model-following controller, respectively. In the meantime, the control effort under the robust adaptive control scheme is much less than that under PID, which is important for prolonging the lifetime of the actuator.
\end{abstract}

Index Terms-Artificial muscles, conjugated polymer actuators, model reduction, physical model, polypyrrole (PPy), robust adaptive control.

\section{INTRODUCTION}

$\mathbf{E}$ LECTROACTIVE polymers (EAPs), also known as artificial muscles, are emerging actuation and sensing materials with numerous potential applications in robotics and biomedical systems [1]-[5]. One class of EAP materials are conjugated polymers, which are also called conducting polymers or synthetic metals [2], [6], [7]. Polypyrrole (PPy) and polyaniline are two of the most commonly used conjugated polymers for actuation purposes. The backbones of conjugated polymers have alternating single and double carbon-carbon bonds (conjugation),

Manuscript received December 4, 2006; revised April 12, 2007. Manuscript received in final form July 19, 2007. Recommended by Associate Editor S. Devasia. This work was supported in part by the National Science Foundation under CAREER Grant ECS 0547131 and by the Michigan State University Intramural Research Grants Program under Grant 05-IRGP-418.

Y. Fang and X. Tan are with the Smart Microsystems Laboratory, Department of Electrical \& Computer Engineering, Michigan State University, East Lansing, MI 48824 USA (e-mail: fangyang@egr.msu.edu; xbtan@msu.edu).

G. Alici is with the School of Mechanical, Materials, and Mechatronics Engineering, University of Wollongong, Wollongong, 2522 NSW, Australia (e-mail: gursel@uow.edu.au).

Digital Object Identifier 10.1109/TCST.2007.912112 which results in positive charge carriers and thus electrical conductivity when electrons are removed from the polymers electrochemically by applying a sufficiently positive potential (oxidation). During oxidation, negatively charged anions are incorporated into the polymer backbone to maintain the charge neutrality. Application of a sufficiently negative potential can reverse the process and reduce the polymer, and the previously trapped anions are repelled out of the polymer. Mass transport of ions and solvent during reduction/oxidation (redox) is considered to be the primary mechanism responsible for volumetric change and thus the actuation capability of conjugated polymers [2]. This can be exploited to create actuators of different configurations including, e.g., bilayer benders [8], trilayer benders [9]-[11], and linear extenders [6], [12]. Conjugated polymer actuators require low actuation voltage (under $1 \mathrm{~V}$ ), generate considerable stress and large strain output, and are light and biocompatible. These advantages make them attractive for a wide range of robotic and biomedical applications, such as micro- and biomanipulation [8], [13], [14], biomimetic systems [15], and biomedical devices [16]-[18].

It is critical to precisely control the force and/or displacement output of conjugated polymer actuators in many of their intended applications such as manipulation of single cells and microsurgical operations. There has been extensive work on understanding the actuation mechanism of conjugated polymers as well as improving their actuation performance (strain output, strain rate, force output, work per cycle, lifetime, etc.) [2], [6], [7], [19]. However, control and control-oriented modeling of conjugated polymers remain largely unexplored. A proportional controller was used by Qi et al. to speed up the transient responses of a polyaniline actuator [20]. Madden treated the actuation dynamics as a first-order system and designed a proportional-integral-derivative (PID) controller for a PPy actuator, where his main interest was to demonstrate a feedback loop consisting of PPy actuator and sensor [21]. Taking again a first-order empirical model, Bowers did simulation studies on PID and adaptive control of conjugated polymers, but no experimental results were presented [22]. The primitive state of conjugated polymer control study is mainly dictated by the sophisticated electrochemomechanical processes during redox reactions, which makes it challenging to have a physical (nonempirical) model suitable for real-time control. In addition, actuator behaviors are difficult to characterize since they are heavily influenced by the materials and processes used during fabrication as well as the environmental conditions (temperature, humidity, electrolyte, etc.) during operation.

The major contribution of this paper is the development of a robust adaptive control scheme for conjugated polymer 
actuators with demonstrated performance in trajectory tracking experiments. A key component of this work is a simple model structure derived from a full, infinite-dimensional physical model through model reduction. Thus, it captures essential actuation dynamics yet is amenable for efficient real-time control. The latter property is of interest since it enables compact, embedded controller implementation for various micro-, robotic, or biomedical applications. While the proposed method applies to general conjugated polymers, a trilayer PPy actuator is used throughout as an example. When operating in air, its actuation behavior shows significant variation over time due to solvent evaporation. This provides an ideal testbed for examining the proposed adaptive scheme. A brief account of the contributions follows.

A low-order model is obtained by reducing the "diffusive-elastic-metal" model presented by Madden [23]. The latter is infinite-dimensional and captures the main electrochemical dynamics during redox processes. Model reduction is achieved by ignoring high-frequency dynamics, which is based on the relatively low operating frequency typically adopted in practice for conjugated polymers. A self-tuning regulator [24] is designed based on the identified parameters to make the closed-loop system follow a reference model. A parameter projection step ensures that the parameter estimates stay within the physically meaningful region, and thus makes the system robust against measurement noises and unmodeled dynamics and nonlinearities.

Tracking experiments are performed to verify the performance of the robust adaptive control scheme. It is shown that, during 4-h continuous operation, the proposed method delivers consistent tracking performance with the normalized tracking error (to be defined later) decreasing from $11 \%$ to $7 \%$, while in comparison, the error increases from $7 \%$ to $28 \%$ and to $50 \%$ under a PID controller and a fixed model-following controller, respectively. In the mean time, the control effort required under the adaptive scheme is much less than that under PID, which is important for extending the lifetime of polymer actuators. Furthermore, the evolution of estimated parameters over time agrees with the model prediction when the diffusion constant decreases due to solvent evaporation, which confirms the capability of the reduced model in capturing underlying physics from a different perspective.

The remainder of this paper is organized as follows. The trilayer PPy actuator and its infinite-dimensional physical model are introduced in Section II. Model reduction is presented in Section III. In Section IV, the design of robust adaptive controller is described. Experimental results are shown in Section V. Finally, concluding remarks are provided in Section VI.

\section{TRILAYER PPy ACTUATOR AND ITS FULL MODEL}

\section{A. Trilayer PPy Actuator}

The trilayer PPy actuator is illustrated in Fig. 1. It is fabricated by the Intelligent Polymer Research Institute, University of Wollongong, Wollongong, NSW, Australia, and a description of the fabrication process can be found in, e.g., [11]. In the middle is an amorphous, porous polyvinylidene fluoride

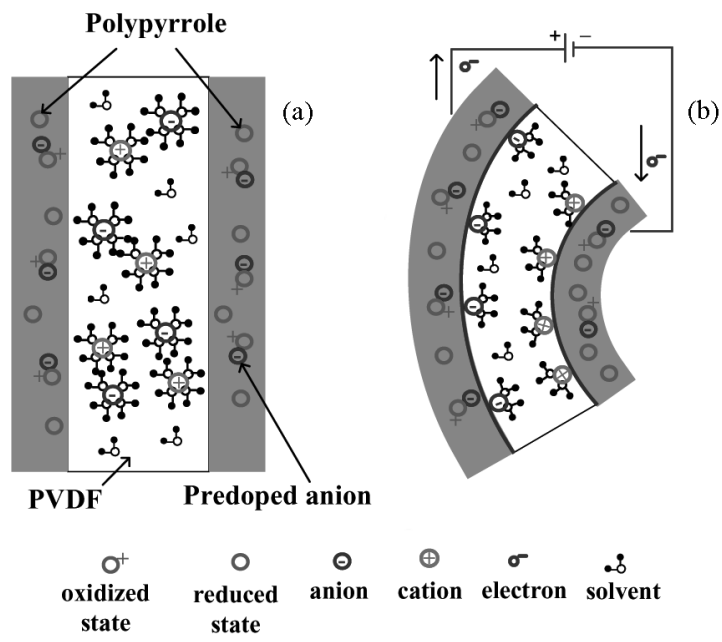

Fig. 1. Illustration of the actuation mechanism of trilayer PPy actuator. (a) Sectional view of the trilayer structure. (b) Bending upon application of a voltage.

(PVDF) layer (110 $\mu \mathrm{m}$ thick) that serves both as a backing material and a storage tank for the electrolyte. The electrolyte used is $0.05 \mathrm{M}$ tetrabutylammonium hexafluorophosphate $\left(\mathrm{TBA}^{+} \mathrm{PF}_{6}^{-}\right)$ in the solvent propylene carbonate (PC). On both sides of the actuator are the PPy layers (30 $\mu \mathrm{m}$ each). During the electrochemical deposition of these layers, the anions $\mathrm{PF}_{6}^{-}$were introduced into the polymer matrix (a process called doping).

When a voltage is applied across the actuator, the PPy layer on the anode side is oxidized while that on the cathode side is reduced. The redox process can be described as

$$
\begin{aligned}
& \text { Oxidation: } \mathrm{PPy}+\mathrm{PF}_{6}^{-} \rightarrow \mathrm{PPy}^{+} \mathrm{PF}_{6}^{-}+e^{-} \\
& \text {Reduction: } \mathrm{PPy}^{+} \mathrm{PF}_{6}^{-}+e^{-} \rightarrow \mathrm{PPy}+\mathrm{PF}_{6}^{-}
\end{aligned}
$$

where PPy represents the neutral state of $\mathrm{PPy}$ and $\mathrm{PPy}^{+}$is the oxidized state; $\mathrm{PPy}^{+} \mathrm{PF}_{6}^{-}$indicates that $\mathrm{PF}_{6}^{-}$is incorporated into the polymer; and $e^{-}$denotes an electron. The oxidized layer absorbs anions and expands as a result, while the reduced layer gives up anions and contracts. The differential expansion thus leads to bending of the actuator, as shown in Fig. 1(a).

\section{B. Electrochemomechanical Model of Trilayer PPy Actuator}

The electrochemomechanical model consists of three modules [25]: 1) the electrical admittance module relating the current (and thus the charge transferred) to the voltage input; 2) the electromechanical coupling module expressing the generated stress in terms of the transferred charge; and 3) the mechanical module connecting the generated stress to the displacement or the force output of the actuator.

1) Electrical Admittance Module: Consider a conjugated polymer film in contact with an electrolyte, as shown in Fig. 2(a). This represents, for instance, one PPy layer in contact with the electrolyte-containing PVDF layer in the case of trilayer PPy actuator. There are two possible mechanisms for the accumulated anions to enter the polymer matrix, diffusion [26], and migration [27], [28]. However, it was shown in [29] and [30] that the migration effect is negligible when the polymer is highly conductive. Madden thus proposed a diffusive-elastic-metal model for PPy, where it was assumed that 


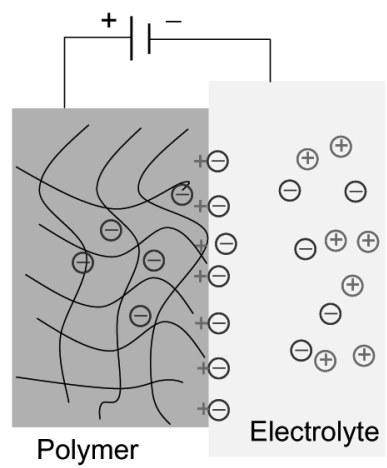

(a)

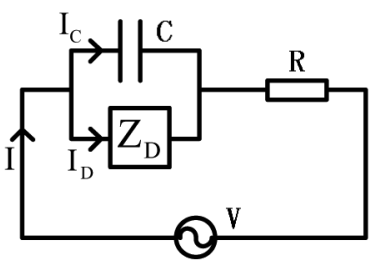

(b)
Fig. 2. (a) Illustration of double-layer charging and diffusion for a conjugated polymer film with one side in contact with electrolyte. (b) Equivalent circuit model for the polymer impedance.

the polymer matrix is perfectly conducting and the ion transport within the polymer is solely determined by diffusion [23]. The admittance model showed good agreement with experimental data over a wide range of frequencies [23], and thus will be adopted in this paper. The model is briefly described next, and we present a different and more straightforward derivation of the final admittance expression than the one given in [23].

Fig. 2(b) shows an equivalent circuit model of the polymer impedance, where $C$ denotes the double-layer capacitance at the polymer/electrolyte interface and $R$ is the electrolyte and contact resistance. $Z_{D}$ represents the "diffusion impedance," which will be clarified in the following derivation. In the Laplace domain, the total current $I(s)$ in the circuit is the sum of the double-layer charging current $I_{C}(s)$ and the current $I_{D}(s)$ diffusing into polymer

$$
I(s)=I_{C}(s)+I_{D}(s) .
$$

The Kirchhoff's voltage law gives

$$
V(s)=I(s) \cdot R+\frac{1}{s \cdot C} \cdot I_{C}(s) .
$$

Let $x$ denote the thickness direction of the polymer, with $x=0$ representing the polymer/electrolyte interface. Assume that the ion concentration varies only in the $x$-direction, which will be denoted as $c(x, s)$. From Fick's law of diffusion, one has

$$
I_{D}(s)=-\left.F \cdot A \cdot D \cdot \frac{\partial c}{\partial x}(x, s)\right|_{x=0}
$$

where $A$ is the surface area of the polymer, $F$ is the Faraday constant, $D$ is the diffusion coefficient, and $(\partial c) /\left.(\partial x)(x, s)\right|_{x=0}$ represents the gradient of ion concentration at the interface. To compute $I_{C}(s)$, one first calculates the charges $Q_{C}(s)$ stored in the double-layer capacitor. Assume that the double layer has a thickness $\delta$ and that the ion concentration within the (thin) double-layer is uniform, which equals $c(0, s)$. Then, $Q_{C}(s)=$ $F \cdot A \cdot \delta \cdot c(0, s)$, which leads to

$$
I_{C}(s)=s \cdot Q_{C}(s)=F \cdot A \cdot \delta \cdot s \cdot c(0, s) .
$$

The diffusion equation in the time domain reads

$$
\frac{\partial c}{\partial t}=D \frac{\partial^{2} c}{\partial x^{2}}, \quad 0<x<h
$$

where $h$ is the thickness of the polymer layer. In the frequency domain, (5) is written as

$$
\frac{s}{D} \cdot c(x, s)=\frac{\partial^{2} c(x, s)}{\partial x^{2}} .
$$

The last equation needed is the boundary condition

$$
\left.\frac{\partial c(x, s)}{\partial x}\right|_{x=h}=0
$$

meaning that there is no ionic flux at $x=h$.

Equations (1)-(4), (6), and (7) form the complete descriptions of the model. Instead of using separation of variables as in [23], a more straightforward model derivation in the frequency domain is provided in the Appendix. It shows that the admittance model of a conjugated polymer with one side in contact with electrolyte is

$$
Y_{1}(s)=\frac{I(s)}{V(s)}=\frac{s\left[\frac{\sqrt{D}}{\delta} \tanh (h \sqrt{s / D})+\sqrt{s}\right]}{\frac{\sqrt{s}}{C}+R s^{3 / 2}+R \frac{\sqrt{D}}{\delta} s \tanh (h \sqrt{s / D})} .
$$

For the trilayer actuator, the voltage input $V(s)$ is applied across two double-layers, and therefore, the admittance $Y(s)$ will be half of (8)

$$
Y(s)=\frac{1}{2} \cdot \frac{s\left[\frac{\sqrt{D}}{\delta} \tanh (h \sqrt{s / D})+\sqrt{s}\right]}{\frac{\sqrt{s}}{C}+R s^{3 / 2}+R \frac{\sqrt{D}}{\delta} s \tanh (h \sqrt{s / D})} .
$$

2) Electromechanical Coupling: The anions transferred to the polymer cause expansion of the polymer. It was shown that the induced in-plane strain $\epsilon$ is proportional to the density $\rho$ of the transferred charges [31]

$$
\epsilon=\alpha \rho
$$

where $\alpha$ is the strain-to-charge ratio. Equivalently, the induced stress by the transferred charges is

$$
\sigma=\alpha E_{\text {ppy }} \rho
$$

where $E_{\text {ppy }}$ denotes the Young's modulus of PPy.

Since the bulk capacitance of the polymer is much larger than the double-layer capacitance, the charges stored in the double layer at the steady state is negligible in comparison with that in the bulk [21]. Consequently, one can obtain the density $\rho(s)$ by

$$
\rho(s)=\frac{I(s)}{s W L h}
$$

where $W$ and $L$ are the width and the length of the PPy layer, respectively.

3) Mechanical Output: Consider a trilayer actuator clamped at one end, as shown in Fig. 3. It can be shown through moment balance that the (uniform) beam curvature $\kappa$ under the induced 


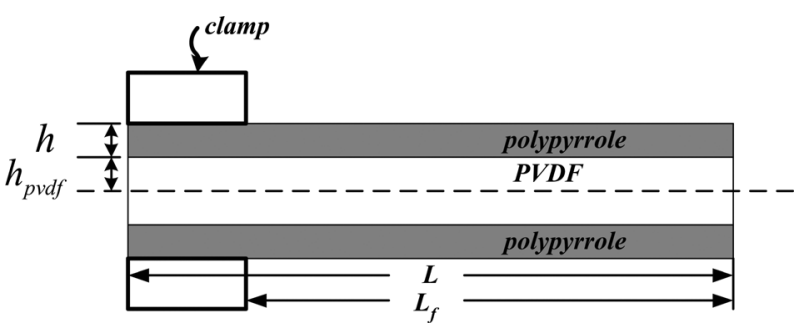

Fig. 3. Geometry of the trilayer actuator.

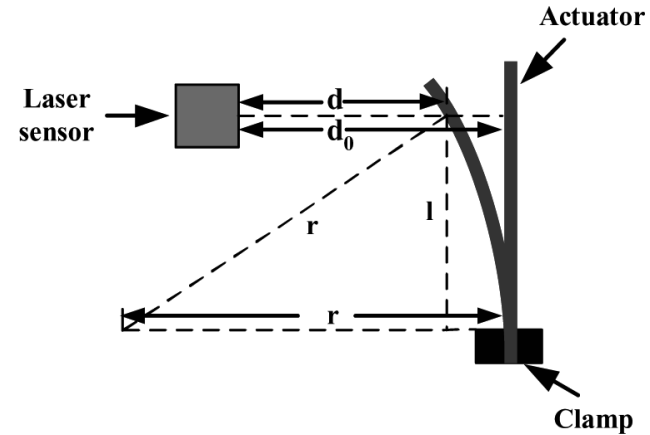

Fig. 4. Geometric relationship between the beam curvature and the tip displacement.

stress (11) and in the absence of external force is [21]

$$
\kappa=\frac{3 \alpha}{2 h_{\mathrm{pvdf}}} \cdot \frac{\left(1+\frac{h}{h_{\mathrm{pvdf}}}\right)^{2}-1}{\left(1+\frac{h}{h_{\mathrm{pvdf}}}\right)^{3}+\frac{E_{\mathrm{pvdf}}}{E_{\mathrm{ppy}}}-1} \cdot \rho
$$

where $E_{\text {pvdf }}$ is the Young's modulus of the PVDF layer, and $h_{\text {pvdf }}$ denotes half of its thickness.

In the experiments, the beam tip displacement is measured by a laser distance sensor, as illustrated in Fig. 4. One can relate the measured displacement $y=d_{0}-d$ to the curvature $\kappa$ via simple geometric calculations

$$
\kappa=\frac{1}{r}=\frac{2 y}{y^{2}+l^{2}}
$$

where $l$ is the distance between the clamped end and the laser incident point when the beam is at rest. For small bending $(y \ll l)$, the curvature is approximately linear with respect to the displacement

$$
\kappa \approx \frac{2 y}{l^{2}}
$$

Combining (9), (12), (13), and (14), one obtains the transfer function from the voltage input $V(s)$ to the bending displacement output $y(s)$

$$
\frac{y(s)}{V(s)}=C_{m} \frac{\frac{\sqrt{D}}{\delta} \tanh \left(h \sqrt{\frac{s}{D}}\right)+\sqrt{s}}{\frac{\sqrt{s}}{C}+R s^{3 / 2}+R \frac{\sqrt{D}}{\delta} s \tanh \left(h \sqrt{\frac{s}{D}}\right)},
$$

with the constant

$$
C_{m} \triangleq \frac{3 \alpha l^{2}\left[\left(1+\frac{h}{h_{\mathrm{pvdf}}}\right)^{2}-1\right]}{8 h_{\mathrm{pvdf}} h W L\left[\left(1+\frac{h}{h_{\mathrm{pvdf}}}\right)^{3}+\frac{E_{\mathrm{pvdf}}}{E_{\mathrm{ppy}}}-1\right]} .
$$

Note that the mechanical dynamics is not considered in the derivation. This is justified by the fact that the actuation frequency is typically under several hertz in practice, which is much lower than the natural frequency of the beam (e.g., typically around $70 \mathrm{~Hz}$ for a sample with dimensions $20 \times 5 \times$ $0.17 \mathrm{~mm}^{3}$ ). The limited actuation frequency is dictated by the relatively low bandwidth of the actuator used in this work. Actuators with higher bandwidth have recently been reported [11], and for those cases, one would need to incorporate appropriate mechanical dynamics into the model.

\section{MODEL REDUCTION}

The full electrochemomechanical model (15) can be rewritten as

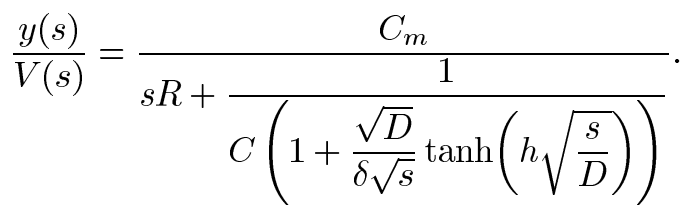

This is an infinite-dimensional system due to the term $\tanh (h \sqrt{(s / D)})$, and thus is not suitable for real-time control purposes. Utilizing the equality [23]

$$
\frac{\tanh \left(\frac{1}{2} \sqrt{\frac{s}{z}}\right)}{4 \sqrt{s z}}=\sum_{n=0}^{\infty} \frac{1}{s+\pi^{2}(2 n+1)^{2} z}
$$

and letting $z=\left(D / 4 h^{2}\right)$, one converts (16) into

$$
=\frac{\frac{y(s)}{V(s)}}{s R+\frac{C_{m}}{C\left(1+\frac{2 D}{h \delta} \sum_{n=0}^{\infty} \frac{1}{s+\pi^{2}(2 n+1)^{2} D(2 h)^{-2}}\right)}} .
$$

To understand the rationale behind model reduction, take typical parameters $D=2 \times 10^{-10} \mathrm{~m}^{2} / \mathrm{s}$ and $h=30 \mu \mathrm{m}$. The constant $\pi^{2} D(2 h)^{-2}=0.548$. The pole of $\left(1 /\left(s+\pi^{2}(2 n+\right.\right.$ $\left.\left.1)^{2} D(2 h)^{-2}\right)\right)$ is at -13.7 for $n=2$, at -26.9 for $n=3$, and at -44.4 for $n=4$, etc. This indicates that one can obtain a low-order approximation to (17) by ignoring terms associated with large $n$. In particular, for a low-frequency input, the approximation will preserve well the behavior of (17). ${ }^{1}$

We thus discard terms with $n \geq 2$. This results in the following third-order system for the actuator:

$$
\frac{y(s)}{V(s)}=\frac{b_{1}^{\prime} s^{2}+b_{2}^{\prime} s+b_{3}^{\prime}}{s^{3}+a_{1}^{\prime} s^{2}+a_{2}^{\prime} s+a_{3}^{\prime}}
$$

${ }^{1}$ It turns out that this will also provide a good approximation to the original dynamics at very high frequencies since both models will behave like $\left(C C_{m} /(1+\right.$ $s R C)$ ). 
where the parameters all have explicit physical meanings

$$
\begin{aligned}
a_{1}^{\prime} & =\frac{4 D}{h \delta}+\frac{5 \pi^{2} D}{2 h^{2}}+\frac{1}{R C} \\
a_{2}^{\prime} & =\frac{5 \pi^{2} D^{2}}{h^{3} \delta}+\frac{9 \pi^{4} D^{2}}{16 h^{4}}+\frac{5 \pi^{2} D}{2 h^{2} R C} \\
a_{3}^{\prime} & =\frac{9 \pi^{4} D^{2}}{16 h^{4} R C} \\
b_{1}^{\prime} & =\frac{C_{m}}{R} \\
b_{2}^{\prime} & =\frac{4 D C_{m}}{h \delta R}+\frac{5 \pi^{2} D C_{m}}{2 h^{2} R} \\
b_{3}^{\prime} & =\frac{5 \pi^{2} D^{2} C_{m}}{h^{3} \delta R}+\frac{9 \pi^{4} D^{2} C_{m}}{16 h^{4} R} .
\end{aligned}
$$

For typical parameters, (18) has one pole and one zero that are located far to the left of the imaginary axis comparing to other poles and zeros, and therefore, the model can be further reduced to second order with one zero, as shown next.

The system (18) has three poles with explicit expressions [32]

$$
\begin{aligned}
& p_{1}=2 \sqrt{-Q} \cos \left(\frac{\theta}{3}\right)-\frac{1}{3} a_{1}^{\prime} \\
& p_{2}=2 \sqrt{-Q} \cos \left(\frac{\theta+2 \pi}{3}\right)-\frac{1}{3} a_{1}^{\prime} \\
& p_{3}=2 \sqrt{-Q} \cos \left(\frac{\theta+4 \pi}{3}\right)-\frac{1}{3} a_{1}^{\prime}
\end{aligned}
$$

where

$$
\begin{aligned}
\theta & =\cos ^{-1}\left(\frac{P}{\sqrt{-Q^{3}}}\right) \\
Q & =\frac{3 a_{2}^{\prime}-a_{1}^{\prime 2}}{9} \\
P & =\frac{9 a_{1}^{\prime} a_{2}^{\prime}-27 a_{3}^{\prime}-2 a_{1}^{\prime 3}}{54} .
\end{aligned}
$$

With typical physical parameters [23], $a_{1}^{\prime}, a_{2}^{\prime}$, and $a_{3}^{\prime}$ are all relatively large numbers $(\gg 1)$. This implies $a_{1}^{\prime 2} \gg 3 a_{2}^{\prime}$ in $Q$, and $2 a_{1}^{\prime 3} \gg 9 a_{1}^{\prime} a_{2}^{\prime}$ and $2 a_{1}^{\prime 3} \gg 27 a_{3}^{\prime}$ in $P$, which leads to

$$
\begin{aligned}
Q & \approx-\frac{a_{1}^{\prime 2}}{9} \\
P & \approx-\frac{a_{1}^{\prime 3}}{27} \\
\sqrt{-Q} & \approx \frac{a_{1}^{\prime}}{3} \\
\theta & \approx \cos ^{-1}\left(-\frac{a_{1}^{\prime 3} / 27}{\sqrt{a_{1}^{\prime 6} / 9^{3}}}\right)=\cos ^{-1}(-1)=\pi .
\end{aligned}
$$

The poles (19)-(21) are thus approximately

$$
\begin{aligned}
& p_{1} \approx 2 \cdot \frac{a_{1}^{\prime}}{3} \cdot \cos \left(\frac{\pi}{3}\right)-\frac{a_{1}^{\prime}}{3}=0 \\
& p_{2} \approx 2 \cdot \frac{a_{1}^{\prime}}{3} \cdot \cos \left(\frac{3 \pi}{3}\right)-\frac{a_{1}^{\prime}}{3}=-a_{1}^{\prime} \\
& p_{3} \approx 2 \cdot \frac{a_{1}^{\prime}}{3} \cdot \cos \left(\frac{5 \pi}{3}\right)-\frac{a_{1}^{\prime}}{3}=0 .
\end{aligned}
$$

Clearly, $\left|p_{2}\right|$ is very large while $\left|p_{1}\right|$ and $\left|p_{3}\right|$ are relatively small.
TABLE I

TYPICAL VALUES OF PARAMETERS

\begin{tabular}{|c|c|}
\hline Parameter & Value \\
\hline $\mathrm{D}$ & $2 \times 10^{-10} \mathrm{~m}^{2} / \mathrm{s}$ \\
\hline $\mathrm{h}$ & $30 \mu \mathrm{m}$ \\
\hline $\mathrm{R}$ & $15 \Omega$ \\
\hline$\delta$ & $25 \mathrm{~nm}$ \\
\hline $\mathrm{C}$ & $5.33 \times 10^{-5} \mathrm{~F}$ \\
\hline
\end{tabular}

The analysis on the zeros is simpler. The zeros of (18) are

$$
\begin{aligned}
& z_{1}=\frac{-b_{2}^{\prime}-\sqrt{b_{2}^{\prime 2}-4 \cdot b_{1}^{\prime} \cdot b_{3}^{\prime}}}{2 b_{1}^{\prime}} \\
& z_{2}=\frac{-b_{2}^{\prime}+\sqrt{b_{2}^{\prime 2}-4 \cdot b_{1}^{\prime} \cdot b_{3}^{\prime}}}{2 b_{1}^{\prime}} .
\end{aligned}
$$

With typical parameters, the following holds:

$$
4 \cdot b_{1}^{\prime} \cdot b_{3}^{\prime} \ll b_{2}^{\prime 2}, \quad b_{2} \gg b_{1}
$$

which implies

$$
\begin{aligned}
& z_{1} \approx-\frac{b_{2}^{\prime}}{b_{1}^{\prime}} \\
& z_{2} \approx 0 .
\end{aligned}
$$

Therefore, $\left|z_{1}\right|$ is very large while $\left|z_{2}\right|$ is relatively small.

A numerical example is provided to illustrate the analysis. Table I lists the typical values for the relevant physical parameters. The corresponding system parameters for (18) are

$$
\begin{array}{ll}
a_{1}^{\prime}=2.32 \times 10^{3} & a_{2}^{\prime}=9.79 \times 10^{3} \quad a_{3}^{\prime}=3.38 \times 10^{3} \\
b_{1}^{\prime}=0.0667 C_{m} & b_{2}^{\prime}=71.48 C_{m} \quad b_{3}^{\prime}=195.1 C_{m} .
\end{array}
$$

The three poles are $-0.38,-2319,-3.84$, and the two zeros are -1069 and -2.74 .

When operating in air, the trilayer actuator will dry up due to solvent evaporation. This implies that the diffusion coefficient $D$ will decay over time. It is thus of interest to see whether the previous analysis on pole/zero locations still holds when $D$ is very small. Fig. 5 shows the ratio

$$
\frac{\min \left(\left|p_{2}\right|,\left|z_{1}\right|\right)}{\max \left(\left|p_{1}\right|,\left|p_{3}\right|,\left|z_{2}\right|\right)}
$$

as a function of $D$, while other parameters are chosen as in Table I. It is clear that even when $D$ is close to 0 , one can safely ignore one pole and one zero of (18).

The final reduced model for the trilayer actuator thus has the following structure:

$$
\frac{y(s)}{V(s)}=\frac{B(s)}{A(s)}=\frac{b_{1} s+b_{2}}{s^{2}+a_{1} s+a_{2}} .
$$

It is expected that despite its simple looking, (24) captures the dominant physics of the actuator within the actuation band- 


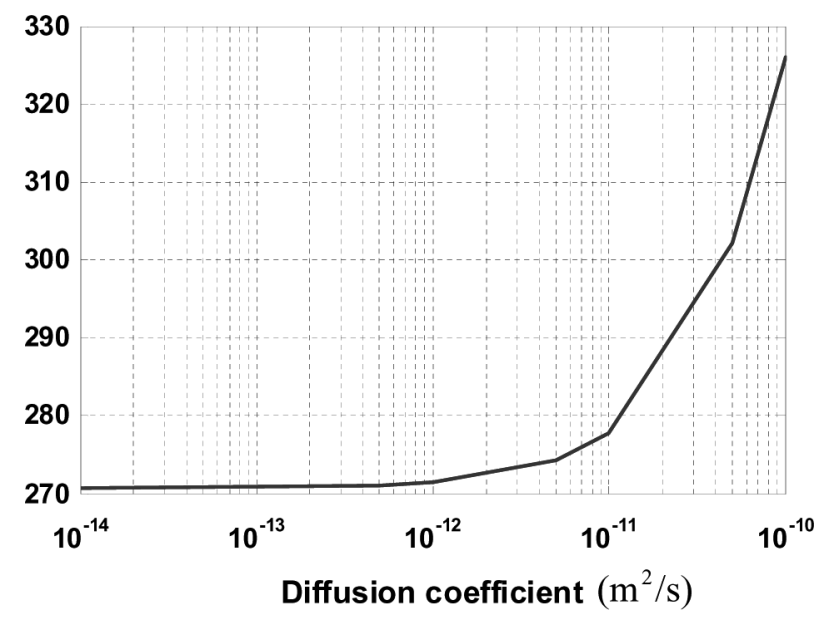

Fig. 5. Ratio $\left(\min \left(\left|p_{2}\right|,\left|z_{1}\right|\right)\right) /\left(\max \left(\left|p_{1}\right|,\left|p_{3}\right|,\left|z_{2}\right|\right)\right)$ as a function of $D$.

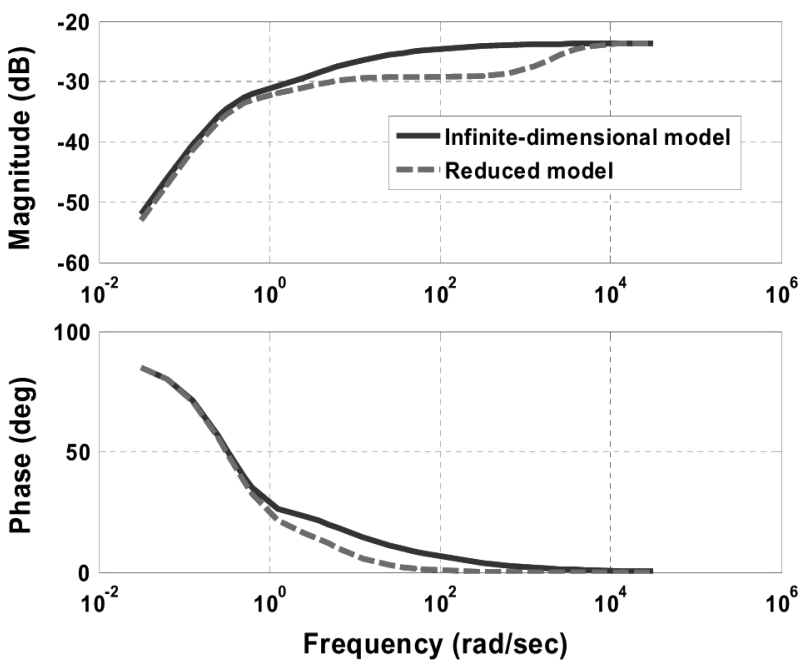

Fig. 6. Comparisons between the infinite-dimensional and reduced model.

width. In particular, all parameters of (24) can be related to fundamental physical parameters for the full model (15).

Fig. 6 compares the Bode plot of (16) and (24) using parameters in Table I. The unmodeled dynamics is bounded, and the maximum discrepancy is reached at around $10^{3} \mathrm{rad} / \mathrm{s}$. Considering that the typical actuation bandwidth is small (several hertz), the reduced model is a good approximation to the full model.

\section{Design OF A Robust AdAPtive CONTROLler}

\section{A. Self-Tuning Regulator}

Controller adaptation is desirable for conjugated polymers since their actuation behaviors can vary significantly over time. Given the model structure (24), various adaptive and robust controllers can be designed [24], [33]. In this paper, a self-tuning regulator is adopted due to its simplicity. The idea is to estimate systems parameters online, and then construct a controller based on these estimates so that the closed-loop system would behave like a model system $G_{m}(s)$ (model following). Fig. 7 illustrates the major components of a self-tuning regulator.

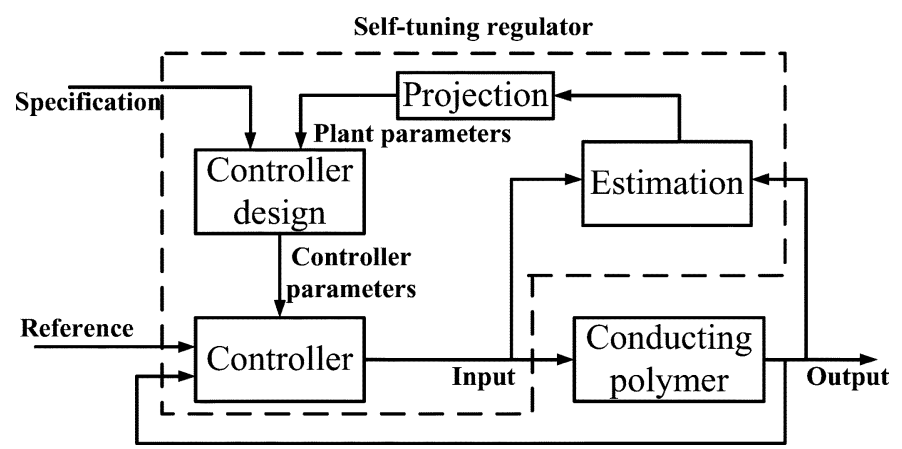

Fig. 7. Illustration of the robust self-tuning regulator.

In the estimation step, the recursive least squares algorithm is chosen to identify parameters in (24) based on the input and output of the conjugated polymer

$$
\begin{aligned}
\frac{d \hat{\theta}}{d t} & =P(t) \varphi(t) e(t) \\
e(t) & =y(t)-\varphi^{T}(t) \hat{\theta}(t) \\
\frac{d P(t)}{d t} & =\beta P(t)-P(t) \varphi(t) \varphi^{T}(t) P(t)
\end{aligned}
$$

where $\beta>0$ is a constant

$$
\begin{aligned}
& \hat{\theta}=\left[\begin{array}{llll}
\hat{a_{1}} & \hat{a_{2}} & \hat{b_{1}} & \hat{b_{2}}
\end{array}\right]^{T} \\
& \varphi^{T}(t)=\left[\begin{array}{lll}
-\frac{d}{d t} \mathcal{L}^{-1}\left(H_{f}(s) y(s)\right) & -\mathcal{L}^{-1}\left(H_{f}(s) y(s)\right)
\end{array}\right. \\
& \left.\times \frac{d}{d t} \mathcal{L}^{-1}\left(H_{f}(s) V(s)\right) \quad \mathcal{L}^{-1}\left(H_{f}(s) V(s)\right)\right] .
\end{aligned}
$$

$\mathcal{L}^{-1}(\cdot)$ denotes the inverse Laplace transform and $H_{f}(s)$ is a filter to avoid direct differentiation of the signals

$$
H_{f}=\frac{1}{s^{2}+\lambda_{1} s+\lambda_{2}}, \quad \lambda_{1}>0, \quad \lambda_{2}>0 .
$$

The desired closed-loop transfer function is chosen to be

$$
G_{m}(s)=\frac{B_{m}(s)}{A_{m}(s)}=\frac{b_{1 m} s+b_{2 m}}{s^{2}+a_{1 m} s+a_{2 m}} .
$$

By solving the Diophantine equation, the controller (30) is obtained to make the closed-loop system follow $G_{m}(s)$

$$
V(s)=\frac{b_{1 m} s+b_{2 m}}{\hat{b}_{1} s+\hat{b}_{2}} r(s)-\frac{\left(a_{1 m}-\hat{a}_{1}\right) s+\left(a_{2 m}-\hat{a}_{2}\right)}{\hat{b}_{1} s+\hat{b}_{2}} y(s)
$$

where $r(s)$ is the reference signal.

\section{B. Parameter Projection}

If the model (24) were exact, the controller (30) in combination with the online parameter identifier would lead to asymptotic model following [24]. However, higher frequency dynamics and nonlinearities are not included in (24). Measurement noises are not reflected in the model either. These undesirable factors might lead to instability of the closed-loop system if no proper steps are taken.

In this paper, parameter projection is adopted as a robustification mechanism for the self-tuning regulator. From the ex- 
pressions of the parameters in (18), one knows that the two poles and the zero of (24) are all negative, thus the parameters $a_{1}, a_{2}, b_{1}$, and $b_{2}$ should all be positive, which are bounded by a small constant $m>0$. Furthermore, based on the given polymer parameters and the knowledge of physical parameters, an upper bound $M>0$ of these parameters can be determined. The update rule (25) is thus modified to incorporate parameter projection shown in (31) at the bottom of the page, where $\hat{\theta}_{i}(t)$ and $[P(t) \varphi(t) e(t)]_{i}$ denote the $i$ th components of $\hat{\theta}$ and $P(t) \varphi(t) e(t)$, respectively. Clearly, if $\hat{\theta}_{i}(0) \in[m, M]$, $\hat{\theta}_{i}(t) \in[m, M], \forall t>0$. In the experiments, the values of $M$ and $m$ are chosen to be $M=1 \times 10^{4}$ and $m=0.001$.

One can represent the true output $y(s)$ of the actuator under the input $V(s)$ as

$y(s)=\frac{b_{1} s+b_{2}}{s^{2}+a_{1} s+a_{2}} V(s)+\Delta(s) V(s)+\mathcal{N}[V(s)]+w(s)$.

Here, $\Delta(s)$ represents the unmodeled higher frequency dynamics, i.e., the difference between (15) and (24). Since both (15) and (24) are stable and have bounded frequency responses, $\Delta(s)$ will be stable and bounded. $\mathcal{N}[V(s)]$ denotes the influence of unmodeled nonlinearity (e.g., hysteresis). The nonlinearities will be bounded due to the dissipative nature of the materials. In (32), w(s) denotes the measurement noise, which is also bounded.

It can be shown [34] that the proposed adaptive control scheme in Section IV-A with parameter projection (31) is robust in the presence of bounded unmodeled dynamics and nonlinearities, measurement noise, and slow and bounded parameter variations; in particular, all signals in the closed-loop system will be bounded.

\section{EXPERIMENTAL RESULTS}

Tracking experiments are conducted to examine the effectiveness of the proposed robust adaptive control scheme. A trilayer PPy actuator $\left(20 \times 5 \times 0.17 \mathrm{~mm}^{3}\right)$ is clamped at one end, where the actuation voltage is applied. The tip displacement is measured by an OADM 20I6441/S14F laser sensor from Baumer Electric, Inc. (Southington, CT) with resolution of $5 \mu \mathrm{m}$. The controller is implemented in a personal computer (PC) equipped with dSPACE DS1104 with sampling frequency set as $1 \mathrm{kHz}$. The experimental setup is shown in Fig. 8. Before each experiment, an actuator cut with the specified size is soaked in the electrolyte ( $\mathrm{TBA}^{+} \mathrm{PF}_{6}^{-}$in propylene carbonate). The inner porous PVDF layer thus stores electrolyte, which enables the actuator to operate in air for some time. The time of continuous in-air operation depends on how fast the stored solvent evaporates, and without further packaging, it is about $4-5 \mathrm{~h}$. The experimental temperature and humidity are maintained as $25^{\circ} \mathrm{C}$ and $27 \%$, respectively. For practical applications, the packaging

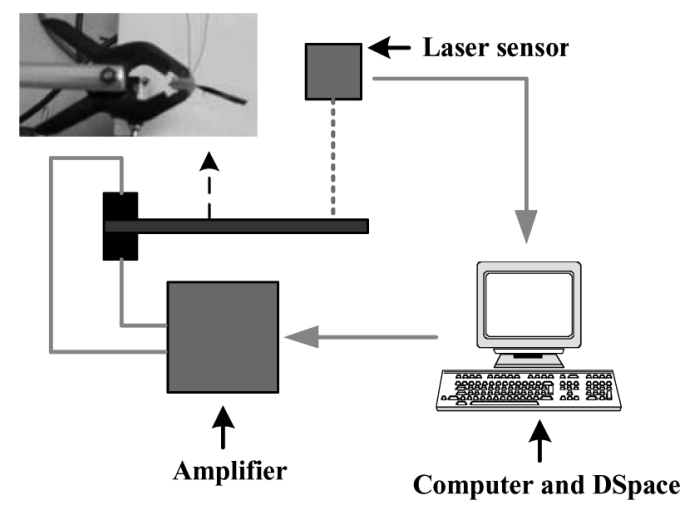

Fig. 8. Schematic of the experimental setup.

issue will have to be solved so that the actuator can work in air for much longer time. On the other hand, the current actuator demonstrates significant time-varying behavior, which provides a good testbed for verifying the proposed adaptive scheme.

For comparison purposes, a PID controller and a fixed modelfollowing controller are also implemented. Actuators with the same dimensions and the same conditions are used for all three controllers. The design of the PID controller and the model-following controller is also based on the model structure (24), while the model parameters are identified in separate experiments shortly before the tracking experiment starts. The modelfollowing controller is constructed as in (30), except that the controller parameters will not be updated. The PID gains are carefully tuned and verified in Matlab simulation before the experiments to ensure that the initial tracking errors are within the similar range as those under other controllers. One example of the PID controller is

$$
\frac{V(s)}{E(s)}=6+\frac{6}{s}+0.02 s
$$

where $E(s)$ is the error signal in frequency domain. For each continuous tracking experiment (3-4 h long), the parameters of the PID controller and the model-following controller remain constant (i.e., nonadapting). Throughout the experiments, the reference model $G_{m}(s)$ is chosen to be

$$
G_{m}(s)=\frac{s+1.5}{s^{2}+4 s+4}
$$

which is based on the desired dynamic responses and actuation constraints of the actuator. Note that a low-pass filter is used to filter the noises in the output signal before the output is sent to the controller. Its bandwidth is chosen to be $30 \mathrm{~Hz}$ to well cover the actuation frequency range in experiments.

In the first batch of experiments (batch one), the reference input $r(t)=0.5 \sin (\pi t)+0.5 \sin (0.2 \pi t) \mathrm{mm}$, and the actuator output $y(t)$ is required to track the desired trajectory $y_{m}(t)=$

$$
\frac{d \hat{\theta}_{i}(t)}{d t}= \begin{cases}0, & \text { if } \hat{\theta}_{i}(t)=M \quad \text { and } \quad[P(t) \varphi(t) e(t)]_{i}>0 \\ 0, & \text { if } \hat{\theta}_{i}(t)=m \text { and } \quad[P(t) \varphi(t) e(t)]_{i}<0 \\ {[P(t) \varphi(t) e(t)]_{i},} & \text { otherwise }\end{cases}
$$




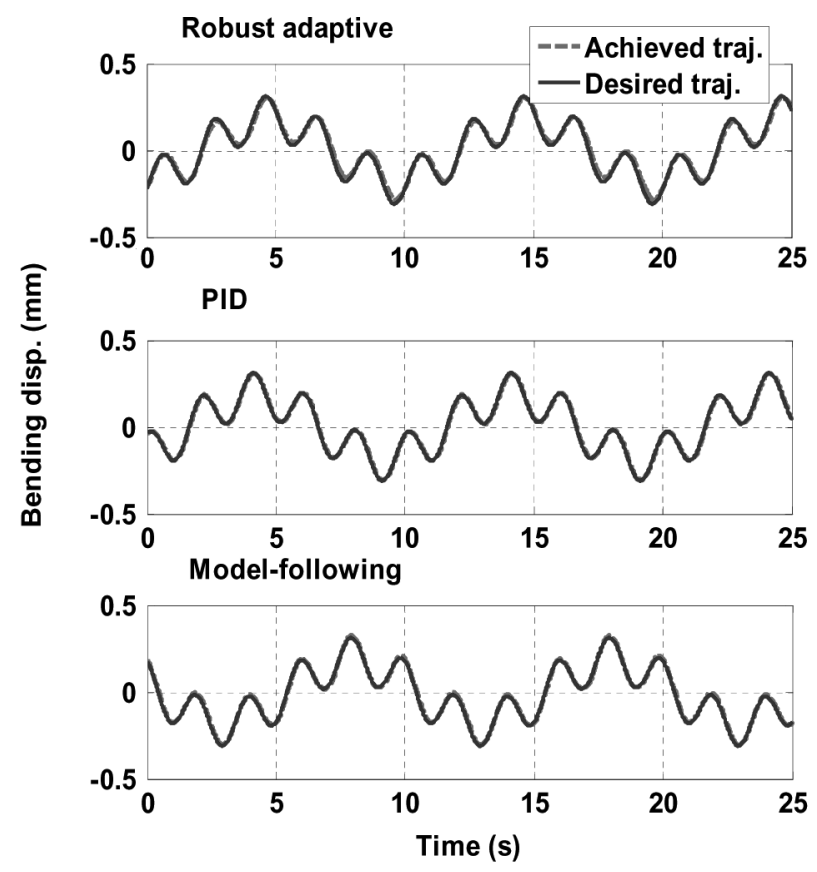

(a)

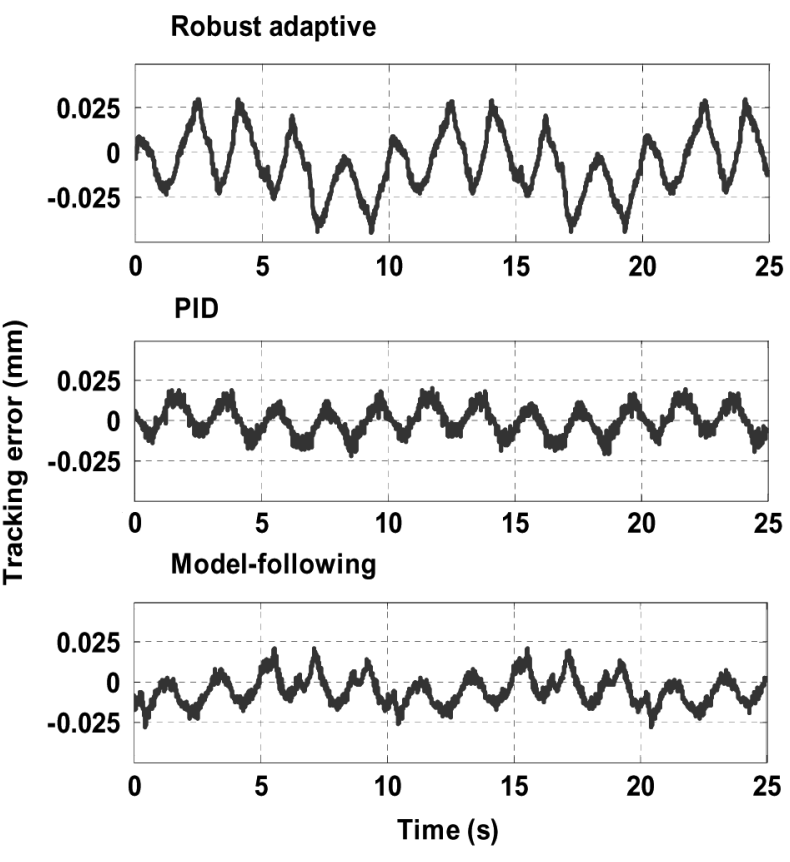

(b)

Fig. 9. Experimental results on trajectory tracking (batch one), $t=0 \mathrm{~h}$. (a) Achieved trajectories versus desired one under the three controllers. (b) Instantaneous tracking errors under the three schemes.

$G_{m}(s)[r(\cdot)](t)$. The latter contains two frequency components $(0.1$ and $0.5 \mathrm{~Hz})$ with peak-to-peak variation of $0.62 \mathrm{~mm}$. Each experiment runs continuously for $4 \mathrm{~h}$. Fig. 9 shows the tracking results at the beginning of the experiment $(t=0 \mathrm{~h})$, while Fig. 10 shows the results when approaching the end of the experiment $(t=4 \mathrm{~h})$.

To better compare the control schemes, two metrics are defined for the tracking error. Given a starting time $t_{0}$ and a constant $T>0$, we define the normalized average error $e_{a}$

$$
e_{a} \triangleq \frac{\int_{t_{0}}^{t_{0}+T}\left|y(t)-y_{m}(t)\right| d t}{\int_{t_{0}}^{t_{0}+T}\left|y_{m}(t)\right| d t}
$$

and the normalized maximum error $e_{m}$

$$
e_{m} \triangleq \frac{\max _{t \in\left[t_{0}, t_{0}+T\right]}\left|y(t)-y_{m}(t)\right|}{\max _{t \in\left[t_{0}, t_{0}+T\right]}\left|y_{m}(t)\right|} .
$$

Throughout this paper, $T$ is chosen to be $100 \mathrm{~s}$. Under the robust adaptive scheme, $e_{a}$ drops from $11 \%$ at $t_{0}=0 \mathrm{~h}$ to $7 \%$ at $t=4$ $\mathrm{h}$, and $e_{m}$ drops from $15 \%$ to $9 \%$ for the same period. In comparison, $e_{a}$ increases from $7 \%$ to $28 \%$ under the PID controller, from $7 \%$ to $50 \%$ under the fixed model-following controller, and $e_{m}$ increases from $8 \%$ to $25 \%$ under the PID scheme, and from $10 \%$ to $48 \%$ under the model-following scheme. Fig. 11 shows the evolution of $e_{a}$ and $e_{m}$, measured and calculated every 30 min, under the three schemes. It is clear that the robust adaptive control scheme delivers consistent tracking performance during the 4-h continuous operation, while the tracking performance under the PID scheme or the fixed model-following scheme deteriorates over time.

A second batch of experiments (batch two) is conducted to examine the effectiveness of the three schemes in tracking trajectories of much larger magnitudes. With the reference input $r(t)=2.1 \sin (0.4 \pi t)+2.2 \sin (0.1 \pi t) \mathrm{mm}$, the desired trajectory $y_{m}(t)$ has peak-to-peak variation of $3 \mathrm{~mm}$. All three schemes show good tracking performance at the beginning. The experiment under PID control has to be stopped after $3 \mathrm{~h}$ since at that time the voltage input exceeds the limit (1.6 V). Fig. 12 shows the tracking results when $t=3 \mathrm{~h}$. Fig. 13 shows the evolution of $e_{a}$ and $e_{m}$. The trend is consistent with that in batch one experiments. It can be seen that the robust adaptive controller keeps $e_{a}$ under 3.5\% and $e_{m}$ under $4 \%$ throughout the 4-h experiment. In the meantime, $e_{a}$ rises from $5.5 \%$ to $8.4 \%$ and $e_{m}$ rises from $5.7 \%$ to $10 \%$, under the PID controller (in $3 \mathrm{~h}$ ); and $e_{a}$ rises from $5 \%$ to $80 \%$ and $e_{m}$ from $5.5 \%$ to $81 \%$, under the fixed model-following controller (in $4 \mathrm{~h}$ ).

It is also important to compare the control efforts required under the different control schemes. Low control effort is highly desirable since that leads to long working life for the conjugated polymer actuator. Fig. 14 shows the evolution of the magnitude of voltage input under each scheme, for both batch one and batch two experiments. The required voltage increases over time under every scheme, which is due to the deteriorating actuation capability of the actuator as the solvent evaporates. However, it can be clearly seen that the voltage input under the adaptive scheme is much lower than that under the PID scheme, and also lower than that under the model-following scheme most of the time. In the batch two/PID experiment, the polymer actuator was actually damaged and stopped functioning after $3 \mathrm{~h}$ due to continuous high-voltage $(>1.5 \mathrm{~V})$ actuation. The reason is that the PID parameters are adjusted based on the system at the beginning of the experiment. However, the system is time varying. Thus, the fixed PID scheme becomes less effective, which tries to reduce the tracking errors by greatly increasing the control voltage amplitude. The fixed model-following scheme uses less control effort than the PID scheme, but it delivers 


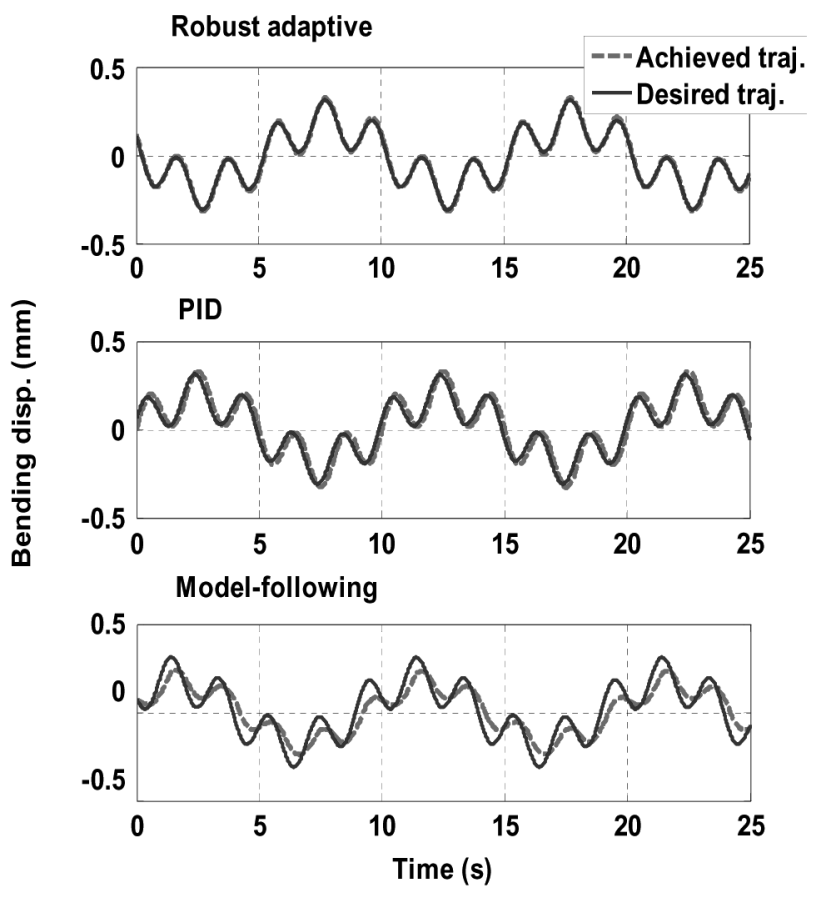

(a)
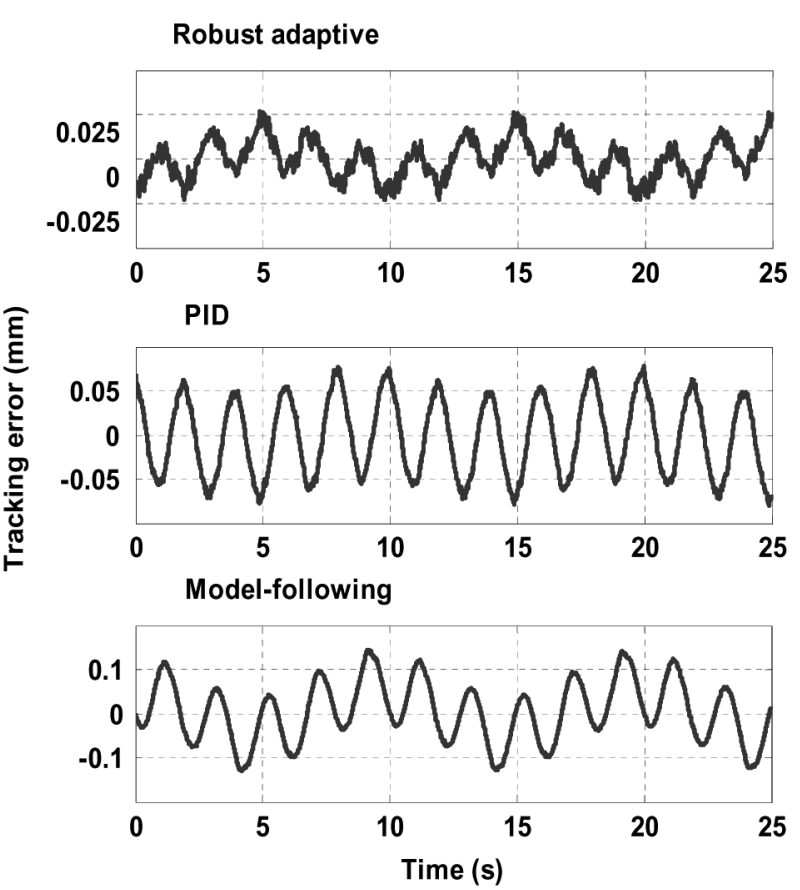

(b)

Fig. 10. Experimental results on trajectory tracking (batch one), $t=4 \mathrm{~h}$. (a) Achieved trajectories versus desired one under the three controllers. (b) Instantaneous tracking errors under the three schemes (note the different vertical-axis scales).

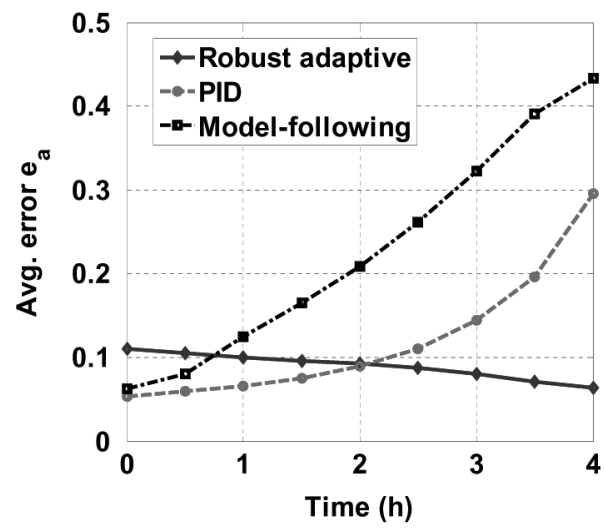

(a)

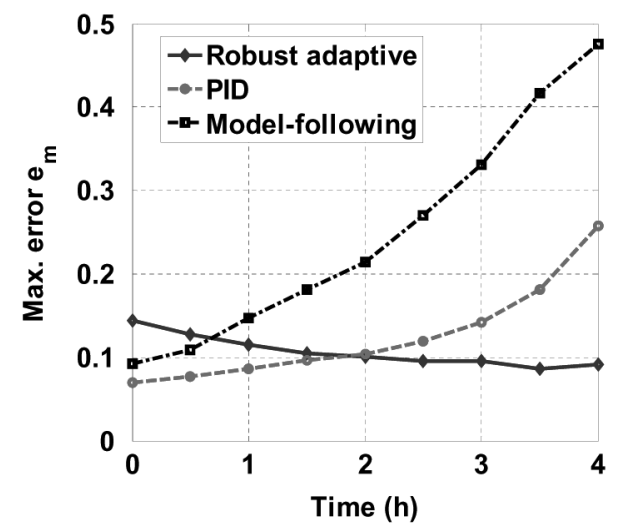

(b)

Fig. 11. Normalized average error $e_{a}$ and maximum error $e_{m}$ under the three control schemes (batch one experiments). (a) Evolution of $e_{a}$. (b) Evolution of $e_{m}$.

the largest error because it does not respond directly to the tracking error.

There is another interesting observation during the experiments that supports the validity of the reduced model. The effect of solvent evaporation can be incorporated by taking the diffusion coefficient $D \rightarrow 0$. This leads to $p_{1} \rightarrow 0, p_{3} \rightarrow 0$, and $z_{2} \rightarrow 0$, where $p_{1}, p_{3}$, and $z_{2}$ are the poles and the zero of the reduced model (24), as defined by (19), (21), and (23). Fig. 15(a) shows the evolution of the poles and the zero during the batch one experiment every $30 \mathrm{~min}$ when the adaptive control scheme is adopted. It can be seen that the poles and zero all tend to 0 , as predicted by the model. Fig. 15(b) also shows a closeup of the evolution during $100 \mathrm{~s}$ after the experiment had been running for $30 \mathrm{~min}$.

In both batch one and batch two experiments, the reference inputs used contain two frequency components and are thus per- sistently exciting of order 4 [24]. This is a necessary and sufficient condition for correctly identifying the four parameters in (24). In practice, however, the persistent excitation condition may not always hold, and it is of interest to know whether the robust adaptive scheme still works well in that case. An experiment is conducted for this purpose, where the reference input $r(t)=2 \sin (\pi t) \mathrm{mm}$ is persistently exciting of order 2 . Fig. 16 shows the tracking errors under the robust adaptive controller, at the beginning and the end of 4-h continuous operation, which demonstrates the capability of the proposed scheme in tracking a nonpersistently exciting signal.

\section{CONCLUSION}

In this paper, a robust adaptive control scheme has been presented for conjugated polymer actuators. The key to the success of this method is a simple actuator model that is reduced from 

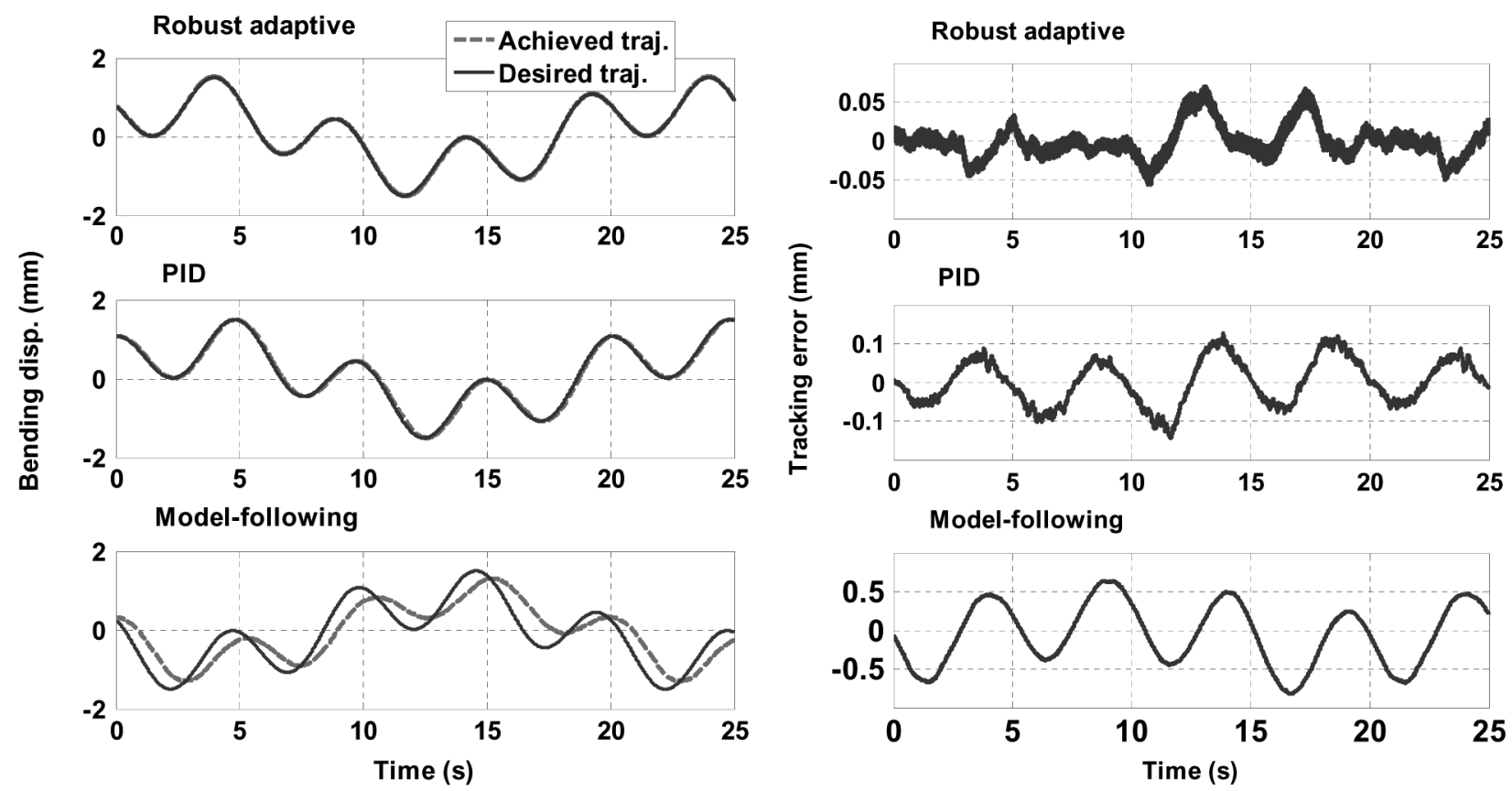

(a)

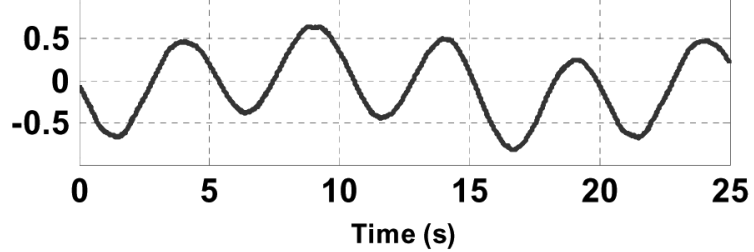

(b)

Fig. 12. Experimental results on trajectory tracking (batch two), $t=3 \mathrm{~h}$. (a) Achieved trajectories versus desired one under the three controllers. (b) Instantaneous tracking errors under the three schemes.

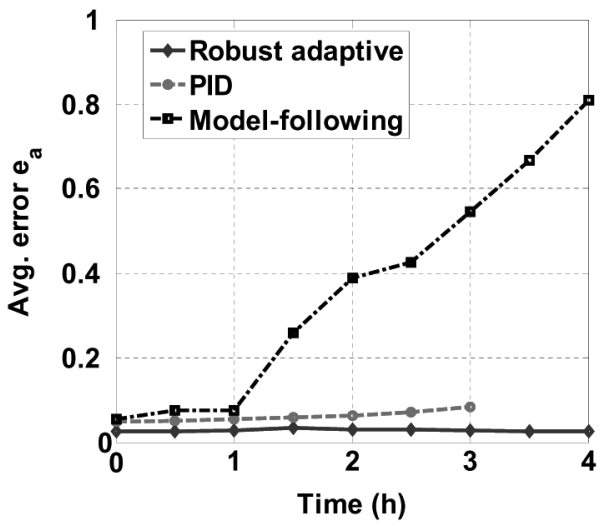

(a)

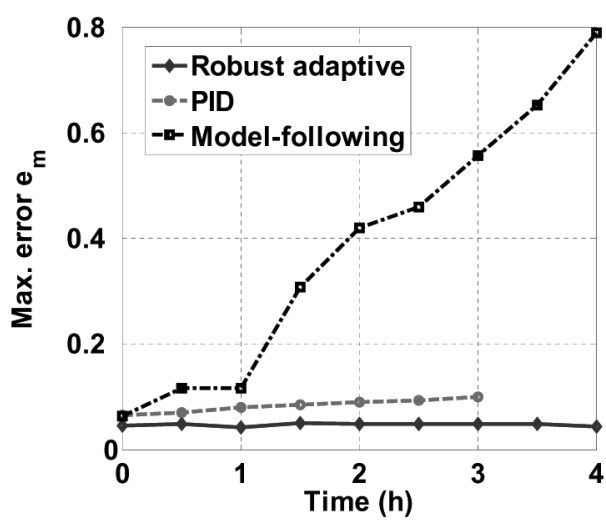

(b)

Fig. 13. Normalized average error $e_{a}$ and maximum error $e_{m}$ under the three control schemes (batch two experiments). (a) Evolution of $e_{a}$. (b) Evolution of $e_{m}$.

the full, infinite-dimensional physical model. Model reduction is based on the knowledge of the actuation bandwidth as well as typical values of the physical parameters. The reduced model captures relevant actuation physics and it makes the control design and implementation easy. Note that the physical-based model provides justification of the specific controller structure. This is in contrast to pure empirical models obtained through system identification, which requires reidentification for different samples. The parameter projection step in the self-tuning regulator ensures the stability of the closed-loop system in the presence of noises and unmodeled dynamics. Experimental results have shown that the proposed scheme is superior to the commonly used PID scheme and to the fixed model-following scheme in terms of both tracking accuracy and required control effort.

The proposed scheme is not restricted to the trilayer PPy actuator although the latter has been used as an example throughout this paper. The model structure applies to other conjugated poly- mers as well as other actuator configurations (such as bilayer or linear actuators). It is not the intention of this paper to just ensure consistent in-air operation of the PPy actuator for $4 \mathrm{~h}$. The significant, relatively fast behavior change of the actuator used here can be thought of as an "accelerated" version of what one might encounter for mature conjugated polymer actuators in the future, and it provides a suitable testbed to compare different control strategies.

Note that the tracking error under the robust adaptive control scheme does not converge to zero, which is due to unmodeled dynamics and nonlinearities, and measurement noises. The error can be reduced, but with increased complexity of the controller. In particular, the effects of nonlinearities (e.g., hysteresis, and oxidation level-dependent material properties) can be modeled and compensated for in the controller design. The impact of unmodeled dynamics can be alleviated by keeping more terms of the series in (17) during model reduction and by including proper mechanical dynamics, which might become necessary 


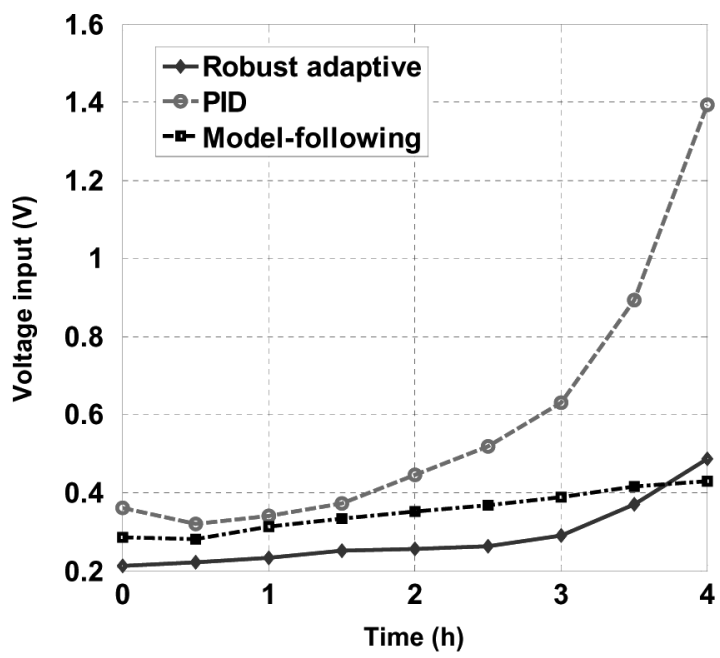

(a)

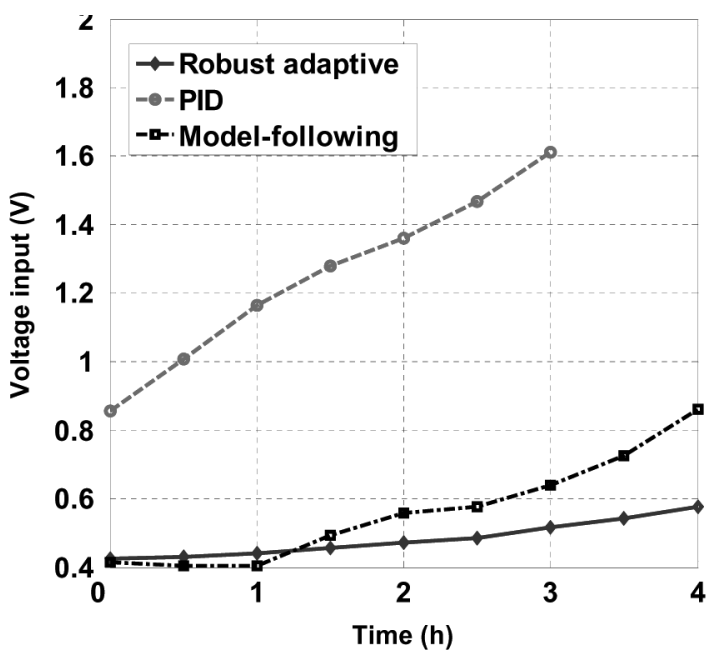

(b)

Fig. 14. Evolution of voltage input magnitude under the three schemes. (a) Batch one experiments. (b) Batch two experiments.

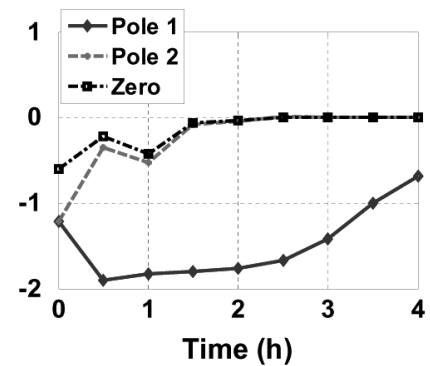

(a)

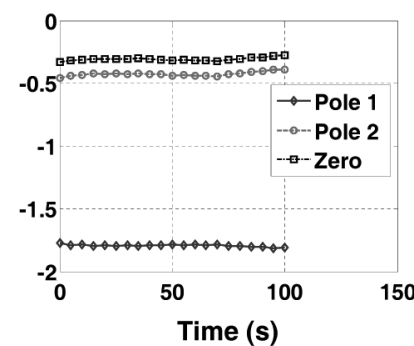

(b)
Fig. 15. Identified poles and zero under the robust adaptive control scheme in batch one experiment. (a) Evolution over 4 h. (b) Evolution over $100 \mathrm{~s}$ (data taken 30 min after the start of experiment).

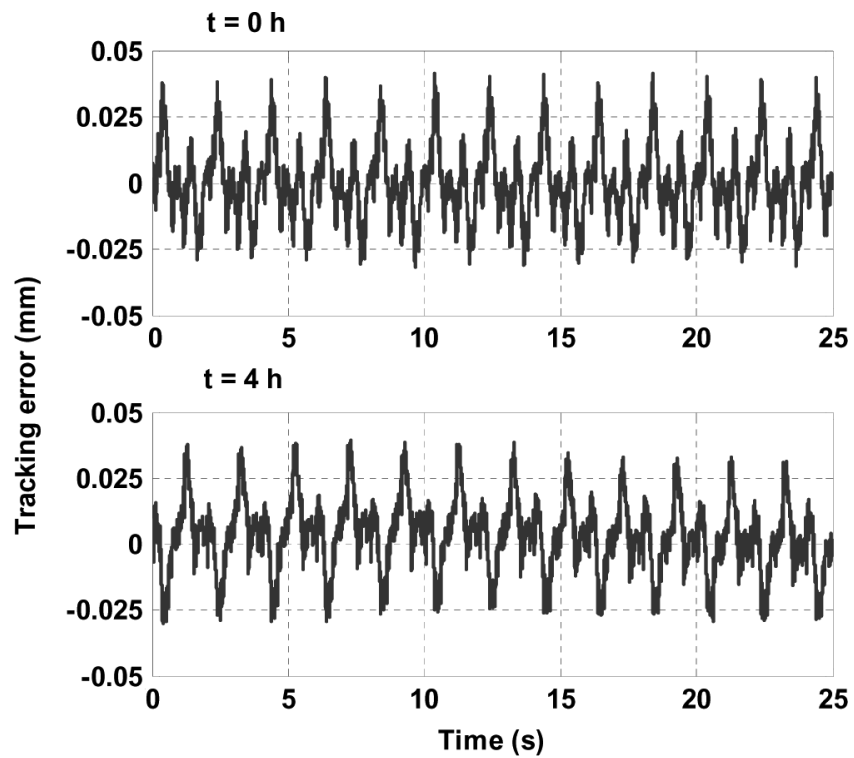

Fig. 16. Experimental results of tracking a nonpersistently exciting signal under the robust adaptive controller: tracking errors at $t=0 \mathrm{~h}$ and $t=4 \mathrm{~h}$.

when operating fast conjugated polymers as those reported in [11].

\section{APPENDIX}

\section{DERIVATION OF THE ELECTRICAL AdMITTANCE MODEL}

Madden used separation of variables to solve the concentration profile in response to a step change of concentration at the interface, and then used convolution of the step response with a given concentration gradient at the boundary to obtain $c(x, t)$ [23]. A more straightforward derivation is given here in the frequency domain. Equation (6) has a generic solution

$$
c(x, s)=C_{1}(s) e^{-\sqrt{s / D} x}+C_{2}(s) e^{\sqrt{s / D} x} .
$$

Letting $x=0$ in (36) and using (4), one gets

$$
C_{1}(s)+C_{2}(s)=\frac{I_{C}(s)}{F \cdot A \cdot \delta \cdot s} .
$$

From (36)

$$
\frac{\partial c(x, s)}{\partial x}=-\sqrt{\frac{s}{D}} C_{1}(s) e^{-\sqrt{s / D} x}+\sqrt{\frac{s}{D}} C_{2}(s) e^{\sqrt{s / D} x} .
$$

Letting $x=0$ in (38) and using (3), one has

$$
-\sqrt{\frac{s}{D}} C_{1}(s)+\sqrt{\frac{s}{D}} C_{2}(s)=-\frac{I_{D}(s)}{F \cdot A \cdot D} .
$$

From (37) and (39), $C_{1}(s)$ and $C_{2}(s)$ can be solved in terms of $I_{C}(s)$ and $I_{D}(s)$

$$
\left\{\begin{array}{l}
C_{1}(s)=\frac{I_{C}(s)}{2 F \cdot A \cdot \delta \cdot s}+\frac{I_{D}(s)}{2 F \cdot A \cdot \sqrt{D \cdot s}} . \\
C_{2}(s)=\frac{I_{C}(s)}{2 F \cdot A \cdot \delta \cdot s}-\frac{I_{D}(s)}{2 F \cdot A \cdot \sqrt{D \cdot s}}
\end{array}\right.
$$

For the trilayer PPy actuator, there is no ionic flux at the other surface of the PPy layer, which gives the boundary condition at $x=h$

$$
\left.\frac{\partial c(x, s)}{\partial x}\right|_{x=h}=0 .
$$


From (1) and (2), $I_{C}(s)$ and $I_{D}(s)$ can be written in terms of $V(s)$ and $I(s)$

$$
\begin{aligned}
& I_{C}(s)=s \cdot C \cdot(V(s)-I(s) \cdot R) \\
& I_{D}(s)=I(s)-s \cdot C \cdot(V(s)-I(s) \cdot R) .
\end{aligned}
$$

Plugging (42) and (43) into (41), one gets the following equation in terms of $V(s)$ and $I(s)$ :

$$
\begin{gathered}
\left(\frac{C}{2 \delta} \frac{e^{\sqrt{s / D} h}-e^{-\sqrt{s / D} h}}{e^{\sqrt{s / D} h}+e^{-\sqrt{s / D} h}}+\frac{C}{2} \sqrt{\frac{s}{D}}\right) V(s) \\
=\left(\frac{R \cdot C}{2 \delta} \frac{e^{\sqrt{s / D} h}-e^{-\sqrt{s / D} h}}{e^{\sqrt{s / D} h}+e^{-\sqrt{s / D} h}}\right. \\
\left.+\left(\frac{1+s \cdot R \cdot C}{2 D}\right) \sqrt{\frac{D}{s}}\right) I(s) .
\end{gathered}
$$

Because

$$
\frac{e^{\sqrt{s / D} h}-e^{-\sqrt{s / D} h}}{e^{\sqrt{s / D} h}+e^{-\sqrt{s / D} h}}=\tanh \left(h \sqrt{\frac{s}{D}}\right)
$$

the admittance model is derived as

$$
\begin{aligned}
Y_{1}(s)= & \frac{I(s)}{V(s)} \\
= & \frac{s\left(\frac{\sqrt{D}}{\delta} \tanh (h \sqrt{s / D})+\sqrt{s}\right)}{\frac{\sqrt{s}}{C}+R s^{3 / 2}+R \frac{\sqrt{D}}{\delta} s \tanh (h \sqrt{s / D})} .
\end{aligned}
$$

\section{REFERENCES}

[1] Y. Bar-Cohen, Ed., Electroactive Polymer (EAP) Actuators as Artificial Muscles: Reality, Potential, and Challenges. Bellingham, WA: SPIE, 2001.

[2] E. Smela, "Conjugated polymer actuators for biomedical applications," J. Adv. Mater., vol. 15, no. 6, pp. 481-494, 2003.

[3] M. Shahinpoor and K. J. Kim, "Ionic polymer-metal composites: IV. Industrial and medical applications," Smart Mater. Structures, vol. 14, pp. 197-214, 2005.

[4] F. Carpi and D. De Rossi, "Electroactive polymer-based devices for e-textiles in biomedicine," IEEE Trans. Inf. Technol. Biomed., vol. 9, no. 3, pp. 295-318, Sep. 2005.

[5] Z. Chen, Y. Shen, N. Xi, and X. Tan, "Integrated sensing for ionic polymer-metal composite actuators using PVDF thin films," Smart Mater. Structures, vol. 16, pp. S262-S271, 2007.

[6] R. Baughman, "Conducting polymer artificial muscles," Synthetic Metals, vol. 78, pp. 339-353, 1996.

[7] G. G. Wallace, G. M. Spinks, L. Kane-Maguire, and P. R. Teasdale, Conductive Electroactive Polymers: Intelligent Materials Systems, 2nd ed. Boca Raton, FL: CRC Press, 2003.

[8] E. Smela, O. Inganas, and I. Lundstrom, "Controlled folding of microsize structures," Science, vol. 268, pp. 1735-1738, 1995.

[9] K. Kaneto, M. Kaneko, Y. Min, and A. G. MacDiarmid, ““'Artificial muscle": Electromechanical actuators using polyaniline films," Synthetic Metals, vol. 71, no. 1-3, pp. 2211-2212, 1995.

[10] G. Alici, B. Mui, and C. Cook, "Bending modeling and its experimental verification for conducting polymer actuators dedicated to manipulation applications," Sens. Actuators A, Phys., vol. 126, pp. 396-404, 2006.

[11] Y. Wu, G. Alici, G. M. Spinks, and G. G. Wallace, "Fast trilayer polypyrrole bending actuators for high speed applications," Synthetic Metals, vol. 156, pp. 1017-1022, 2006.
[12] A. D. Santa, D. De Rossi, and A. Mazzoldi, "Characterization and modelling of a conducting polymer muscle-like linear actuator," Smart Mater. Structures, vol. 6, pp. 23-34, 1997.

[13] E. W. H. Jager, O. Inganas, and I. Lunstrom, "Microrobots for micrometer-size objects in aqueous media: Potential tools for single cell manipulation," Science, vol. 288, pp. 2335-2338, 2000.

[14] J. W. L. Zhou, H.-Y. Chan, T. K. H. To, K. W. C. Lai, and W. J. $\mathrm{Li}$, "Polymer MEMS actuators for underwater micromanipulation," IEEE/ASME Trans. Mechatronics, vol. 9, no. 2, pp. 334-342, Jun. 2004.

[15] P. Madden, J. Madden, P. Anquetil, H. Yu, T. Swager, and I Hunter, "Conducting polymers as building blocks for biomimetic systems," in Proc. Bio-Robot. Symp., Durham, NH, 2001, pp. 1949-1954.

[16] A. Mazzoldi and D. De Rossi, "Conductive-polymer-based structures for a steerable catheter," in Smart Structures and Materials 2000: Electroactive Polymer Actuators and Devices, Y. Bar-Cohen, Ed. Bellingham, WA: SPIE, 2000, pp. 273-280.

[17] C. Immerstrand, K. H. Peterson, K.-E. Magnusson, E. W. H. Jager, M. Krogh, M. Skoglund, A. Selbing, and O. Inganas, "Conjugatedpolymer micro- and milliactuators for biological applications," Mater. Res. Soc. Bull., vol. 27, no. 6, pp. 461-464, 2002.

[18] M. Manto, M. Topping, M. Soede, J. Sanchez-Lacuesta, W. Harwin, J. Pons, J. Williams, S. Skaarup, and L. Normie, "Dynamically responsive intervention for tremor suppression," IEEE Eng. Med. Biol. Mag., vol. 22, no. 3, pp. 120-132, May-Jun. 2003.

[19] P. G. A. Madden, J. D. W. Madden, P. A. Anquetil, N. A. Vandesteeg, and I. W. Hunter, "The relation of conducting polymer actuator material properties to performance," IEEE J. Ocean. Eng., vol. 29, no. 3, pp. 696-705, Jul. 2004

[20] B. Qi, W. Lu, and B. R. Mattes, "Control system for conducting polymer actuators," in Smart Structures and Materials 2002: Electroactive Polymer Actuators and Devices (EAPAD), Y. Bar-Cohen, Ed. Bellingham, WA: SPIE, 2002, pp. 359-366.

[21] P. G. A. Madden, "Development and modeling of conducting polymer atuators and the fabrication of a conducting polymer based feedback loop," Ph.D. dissertation, Dept. Mech. Eng., Massachusetts Inst. Technol., Cambridge, MA, 2003.

[22] T. A. Bowers, "Modeling, simulation, and control of a polypyrrolebased conducting polymer actuator," M. S. thesis, Dept. Mech. Eng., Massachusetts Inst. Technol., Cambridge, MA, 2004.

[23] J. D. W. Madden, "Conducting polymer actuators," Ph.D. dissertation, Dept. Mech. Eng., Massachusetts Inst. Technol., Cambridge, MA, 2000.

[24] K. J. Astrom and B. Wittenmark, Adaptive Control, 2nd ed. Reading, MA: Addison-Wesley, 1995.

[25] Y. Fang, X. Tan, Y. Shen, N. Xi, and G. Alici, "A scalable model for trilayer conjugated polymer actuators and its experimental validation," Mater. Sci. Eng., C, Biomim. Supramol. Syst., vol. 28, pp. 421-428, 2007.

[26] R. Penner, L. Van Dyke, and C. Martin, "Electrochemical evaluation of charge-transport rates in polypyrrole," J. Phys. Chem., vol. 92, pp. 5274-5282, 1988.

[27] R. Bull, F.-R. Fan, and A. Bard, "Polymer films on electrodes," J. Electrochem. Soc., vol. 129, no. 5, pp. 1009-1015, 1982.

[28] H. Mao, J. Ochmanska, C. D. Paulse, and P. G. Pickup, "Ion transport in pyrrole-based polymer films," Faraday Discussions Chem. Soc., vol. 88, pp. 165-176, 1989.

[29] P. Daum, J. R. Lenhard, D. Rolison, and R. W. Murray, "Diffusiona charge transport through ultrathin films of radiofrequency plasma polymerized vinylferrocene at low temperature," J. Amer. Chem. Soc., vol. 102 , pp. 4649-4653, 1980.

[30] T. Amemiya, K. Hashimoto, and A. Fujishima, "Frequency-resolved faradaic processes in polypyrrole films observed by electromodulation techniques: Electrochemical impedance and color impedance spectroscopies," J. Phys. Chem., vol. 97, pp. 4187-4191, 1993.

[31] T. F. Otero and J. M. Sansinena, "Bilayer dimensions and movemen in artificial muscles," Bioelectrochem. Bioenergetics, vol. 42, no. 2, pp. 117-122, 1997.

[32] W. Dunham, Journey Through Genius: The Great Theorems of Mathematics. New York: Wiley, 1990.

[33] S. Boyd, L. El Ghaoui, E. Feron, and V. Balakrishnan, Linear Matrix Inequalities in System and Control Theory. Philadelphia, PA: SIAM, 1994.

[34] S. M. Naik and P. R. Kumar, "Robust indirect adaptive control of timevarying plants with unmodeled dynamics and disturbances," SIAM J. Control Optim., vol. 32, no. 6, pp. 1696-1725, 1994. 


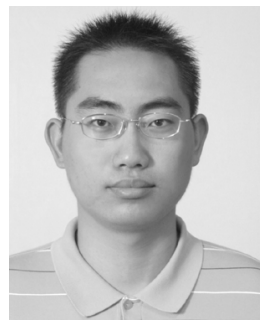

Yang Fang ( $S^{\prime} 07$ ) received the B.S. degree in automatic control from University of Science and Technology of China, Hefei, China, in 2003, and the M.S. degree in manufacturing system and technology from Nanyang Technological University, Singapore, in 2005. Since 2005, he has been working towards the Ph.D. degree in the Electrical and Computer Engineering Department, Michigan State University, East Lansing. His Ph.D. dissertation is focused on the modeling and control of conjugated polymer actuators, modeling of conjugated polymer sensors, and bio/microapplications of these materials.

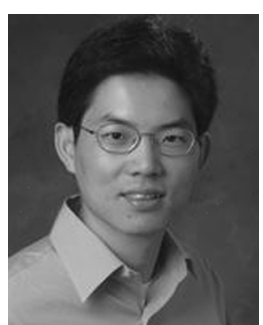

Xiaobo Tan (S'97-M'02) received the B.S. and M.S. degrees in automatic control from Tsinghua University, Beijing, China, in 1995 and 1998, respectively, and the Ph.D. degree in electrical and computer engineering from the University of Maryland at College Park, in 2002.

From September 2002 to July 2004, he was a Research Associate with the Institute for Systems Research (ISR), University of Maryland. In August 2004, he joined the Department of Electrical and Computer Engineering, Michigan State University, East Lansing, as an Assistant Professor. His research interests include modeling and control of smart materials and microelectromechanical systems, electroactive polymer sensors and actuators, biomimetic robotics, bio/micromanipulation, and distributed control of autonomous swarms.

Dr. Tan was an ISR Systems Fellow from 1998 to 2002. He was a finalist for the Best Student Paper Award at the 2002 IEEE Conference on Decision and Control and a recipient of the National Science Foundation (NSF) CAREER Award in 2006.

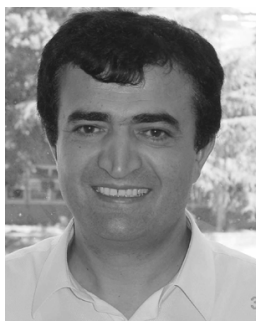

Gürsel Alici received the B.Sc. degree (with high honors) from the Middle East Technical University, Gaziantep, Turkey, in 1988, and the M.Sc. degree from Gaziantep University, Gaziantep, Turkey, in 1990, both in mechanical engineering, and the Ph.D. degree in robotics from Oxford University, Oxford, U.K., in 1993.

Currently, he is an Associate Professor at the School of Mechanical, Materials and Mechatronic Engineering, University of Wollongong, NSW, Australia, where he leads Mechatronic Engineering. He has authored or coauthored more than 100 papers published in international journals and conference proceedings. His current research interests include mechanics, optimum design, control, and calibration of mechanisms/robot manipulators/parallel manipulators, micro/nanorobotic manipulation systems, and modeling, analysis, and characterization of conducting polymer actuators and sensors for use in functional robotic devices. 